In the fourth place, the functional disturbances produced by disease of the gland are discussed. Marie called attention to the association of acromegaly with tumours of this gland, and subsequent writers have mentioned numerous metabolic disorder's associated with such disease, e.g. glycosuria, polyuria, impotence or amenorrhœe, delay in the appearance of secondary sexual characteristics in the young, accompanied by adiposity, subnormal temperature, slowing of pulse, loss of hair, \&c.

The author, in conclusion, summarises the functions of the gland as follows:-It may be regarded as having a double function. The anterior lobe secretion passes into the blood sinuses which traverse this part of the gland, while the hyaline substance, apparently the product of secretion from the epithelial investment of the posterior lobe, enters the cerebro-spinal spaces by way of channels in the pars nervosa (Herring). The latter, although possessing a physiological active principle, as shown by the resuits of injection, does not appear so essential for metabolism as the anterior lobe, the total removal of the latter leading to a very distinctive train of symptoms both in the young and adult animal. The effects of tumours and disease of the gland are most readily interpreted as due to interference with a glandular secretion. It is just possible that certain of the symptoms of disease of the pituitary are due to secondary changes in other glands. These secondary changes are more widespread in pituitary disease than in affections of any other ductless glands, showing how vitally important this ductless gland is in the body economy.

\title{
PHARMACOLOGY AND THERAPEUTICS.
}

By FRANCIS D. BOYD, C.M.G., M.D.

\section{Magnesium Sulphate.}

During the last few years Meltzer and his co-workers have contributed a number of papers on the pharmacological action of magnesium, calcium and potassium. At first of abstract pharmacological interest, the results have now come to be of definite therapeutic value. The pharmacological experiments were carried out on frogs, rabbits, monkeys, and finally applied to the human subject. In the first group of experiments a magnesium salt in isotonic solution was introduced intravenously, subcutaneously, and intra-muscularly. However administered, the pharmacological action was the same-the same phenomena followed the injection. When introduced in small but toxic doses there followed a paralysis of all voluntary movements, a diminution of the reflexes and anæs- 
thesia, but respiration remained unaffected. In lethal doses, however, death occurred from respiratory failure. For rabbits 1.5 to 1.75 grms. per kilo of body weight proved an anæsthetic dose. After such a dose the animal remained in a state of deep sleep and complete muscle relaxation for hours. Waking was abrupt. When the solution of a magnesium salt was concentrated or was rapidly introduced into the circulation, the heart was stopped before it could convey the salt to the muscles or central nervous system. The magnesium action could only be obtained after mouth administration if a previous double nephrectomy had been performed. Direct application of a weak solution of magnesium sulphate to the medulla produced complete paralysis, the animal holding itself "as if its body and head were separate."

Spinal injections of magnesium salts were carried out on apes and on man. On the injection of a medium dose sensibility and motility became completely suspended for some hours. With a larger dose there followed complete unconsciousness, but the respiration was sufficient. Still larger doses produced death from respiratory failure, but death could be averted by artificial respiration. No danger to the circulation was noted from this method of administration. In man it was demonstrated that the subcutaneous injection of magnesium sulphate was followed by deep narcosis lasting some hours; consciousness and sensibility to pain completely disappeared, but not all movement. The injection was in fact not accompanied by any curara-like action. Calcium is directly antagonistic to magnesium. This can be illustrated by a striking experiment. If a rabbit be injected subcutaneously with, for every kilo of weight, about $7 \mathrm{c.cm}$. of a 25 per cent. solution of magnesium sulphate, it will become completely anæsthetised and paralysed. If now one injects into a vein 6 to 8 c.cm. of a 3 per cent. calcium chloride solution, in a short time there is a very striking change. The breathing, which was shallow, becomes deeper and quicker, the lid reflex returns, the paralysed animal makes an effort to turn round, often even before the calcium injection is fully administered. The natural position is rapidly assumed, and the animal, a few minutes before at the point of death, will hop away if its tail be nipped. This rapid restoration will take place even after respiration has ceased, provided the heart is still beating. Such a rapid antagonistic action is almost unique in pharmacological literature.

The very striking results obtained by Meltzer and his colleagues have been now utilised therapeutically. The unsatisfactory 
results obtained in the treatment of tetanus and the apparent uselessness of anti-tetanic serum have long been a favourite theme with the anti-vivisectionists. Phillips, in an interesting paper contributed to the Royal Society of Medicine, discusses the treatment of tetanus by the intra-spinal injection of solution of magnesium sulphate. In the Government Hospital, Cairo, during three years, 1906 to 1908 , twenty-nine cases of tetanus were admitted and twenty-three died, giving a mortality of 75.8 per cent. The disease is common and very fatal amongst native Egyptians, notwithstanding the use of antitoxin. Since the introduction of the magnesium sulphate injections seven cases have been treated and three proved fatal. All the fatal cases were extremely acute. Taken with other reported cases, we get a total of twenty-eight with twelve deaths, a mortality of $42 \cdot 8$ per cent., a very distinct improvement on former figures. The technique of the treatment is simple. A 25 per cent. solution of magnesium sulphate is sterilised and injected into the lumbar spinal meninges, allowing first a little cerebro-spinal fluid to escape. It may be necessary to administer chloroform to abolish oposthotonos before the injection can be carried out. The patient should be propped up during the injection to prevent the fluid extending to the medullary region, as respiratory paralysis might occur. The dosage of the magnesium sulphate solution is $1 \mathrm{c.cm}$. for every $25 \mathrm{lbs}$. of body weight, roughly speaking 5 c.c. to 6 c.cm. for an adult, 2 c.c. to 3 c.c. for a child. The injections should be repeated as necessary. The action of the injections is entirely in the relief afforded by the diminution of spasm and diminished rigidity. The results, so far, are very encouraging, and justify a wider use of the treatment.

Marinesco reports the treatment of four cases of Sydenham's chorea with magnesium sulphate injections, and found that they exercised a remarkable sedative effect on choreic movements, and that by this treatment the disease completely disappears after a few days, and that the results are superior to those obtained by any other method of treatment.

\section{Digitalis.}

At the present time when so much is heard of the physiological standardisation of medicinal substances, a communication by Chevalier to the Therapeutic Society of Paris on the method of Focke for the standardisation of the galenical preparations 
of digitalis seems of interest. Focke's method is very widely used by manufacturing chemists. It consists in the injection into the lymph sac of a frog of a quantity of a one-tenth infusion of the leaves of digitalis sufficient to arrest the heart in seven to twenty minutes. The time of cardiac arrest is noted. A formula is thus obtained

$$
\mathrm{V}=\frac{p}{d \times t}
$$

$p=$ the weight of the frog in grammes, $t=$ the number of minutes required for arrest of the heart, $d=$ the dose of infusion injected in c.cm. For example say a frog weighing 30 grms. shows arrest in twenty minutes with a dose of 0.3 c.c. infusion then

$$
\mathrm{V}=\frac{30}{20 \times 0 \cdot 3}=5 \text {. }
$$

The value or $\mathrm{V}$ of the digitalis leaves would thus be five, and Focke estimates that a good sampl: of lezves should show a value somewhere $\mathrm{n}$ ar five.

Chevalier casts grave doubts on the accuracy and c nsequent value of the method, and in his contention he is supported in a communication by Wiki of Geneva. Both find that even with the strictest precaut ons as regards selected time of observation and variety of frog, there will be marked variations in results. Given the same weight of frog and the same preparation of digitalis, there will be marked variations in the time of production of car diac arrest. Judging from a large number of experiments by both observers the frog'; individuality seems the predominating factor. Wiki "continues to believe that the frog, unless disciplined like a Prussian, is a deplorable 'mêtre' in pharmacology. He shows well the kind but not the quantity of a pharmacol gical action.'

Gunn still further liscredits the frog hitherto so useful to the pharmacologist. In investigating the influence of certain alkaloids upon the peripheral ves els, he finds essential differences between the blood-vessels of the frog and the blood-vessels of mammals in their reaction to ertain alkaloids. Three alkaloids-apocodeine, quinine and yohimbine-which have been shown by other observers to dilate the blood-vessels when perfused through the surviving organs of mammals, contract the blood-vessels of the frog. The rule, therefore, that the peripheral action of a pharmacological agent on the blood-vessels of the frog is qualitatively similar to its peripheral action on the blood-vessels of mammals is not 


\section{Pharmacology and Therapeutics}

invariable. Hence deductions cannot be drawn as to effects of substances on the blood-vessels of mammals based only on experiments on frogs.

The pharmacological action of digitalis has been the subject of a somewhat warm discussion. Etienne, discussing digitalis preparations and their derivatives, concludes that all the preparations and derivatives of digitalis, as far as their action on the rhythm of the heart is concerned, give at first an infrequency of the pulse, which is followed, if the dose be lethal, by an acceleration of the cardiac beat with diverse irregularities. Digalin gives less intense inhibition than the others. The fluid extract, digitonin and digitalin, are most energetic in their inhibitory action; the infusion was found very variable. Etienne concludes that with the modification of the pulse rhythm there is paralysis of the vagus, either central or peripheral, and as digitalis, in whatever form employed, shows itself incapable of inhibiting the heart in the vagotomised dog, one must conclude that inhibition is due to a central action and must exclude the peripheral nerve mechanism. Kochmann deals at length with Etienne's observations, especially criticising the dose employed, and the absence of control observations with vagal stimulation and with the use of atropine. It must be admitted that Kochmann's criticism of Etienne's work is just, and that we can still hold that much of the slowing of the cardiac rhythm after the use of digitalis in therapeutic doses is due to peripheral vagal stimulation, though central action may have a certain influence.

\section{ERgot.}

There is probably no substance in pharmacy and pharmacology around which there is more confusion and contradiction than can be claimed for the literature and teaching of ergot. A medicinal substance of prime importance to the clinician, yet the chemist and pharmacologist have still much to clear up in its chemistry and action.

Cronyn and Henderson, discussing ergot, come to certain conclusions which are of interest to the clinician. They find that the galenical preparations contain considerable amounts of the active principles, but do not show any great or marked action when given by the mouth. Ergotoxine, which has been separated, is a highly active alkaloid, and has the properties most desired in medicine. It brings about a long enduring contraction of the walls of the arterioles and increases the movements of the uterus. This action, however, is feeble when it is administered by the 
mouth, but more marked on subcutaneous injection and much more marked when given intravenously. They advise its use in shock or post-partum hæmorrhage, but here, as a rapid action is desired, it should be administered intravenously. In hæmorrhage from the pulmonary vessels, the administration of ergot seems irrational. No doubt the initial vascular contraction is manifested in the pulmonary vessels, but the maximum vascular constriction is in the splanchnic area, and there is therefore a rise in pressure in the pulmonary vessels and a passive congestion of the lungs. Therefore the use of ergot in hæmoptysis is irrational. The same reasoning applies to hæmorrhage from the intestinal tract.

References.-Meltzer, Deutsch. med. Wochenschr., No. 45, 1909, S. 1963. Phillips, Proceedings of the Royal Society of Medicine, Medical Section, 25th January 1910, p. 39. Marinesco, La Semaine Médicale, 18th November 1908. Chevalier, Bulletin Générale de Thérapeutique, March 1910. Etienne, Archives de Pharmocodynamic, xix. 1909, p. 119. Gunn, Ibid., p. 319. Kochmann, Ibid., p. 327. Cronyn and Henderson, Journal of Pharmacology and Experimental Therapeutics, vol. i. No. 2, p. 202.

\section{NEW BOOKS AND NEW EDITIONS.}

Surgery: Its Principles and Practice. By various Authors. Edited by William Williams Keen and John Chalmers da Costa. Vol: V. Philadelphia and London: W. B. Saunders Co. 1909. Price 30s.

IN issuing the last volume of this work the editors state that the original promise of five volumes of 800 pages each, 4000 pages in all, has been more than fulfilled, for the completed work covers nearly 5500 pages.

The first chapter on the Surgery of the Vascular System is by Matas of New Orleans, who is so well known through having put the operative treatment of aneurysm on a new basis. He discusses the surgery of the pericardium, the heart, and the peripheral vessels. The surgery of the arteries is a subject in which American surgeons have done a great deal of important pioneer work. Readers belonging to the Edinburgh school will be gratified to find the work of Syme and Annandale abundantly recognised. Injuries of vessels produced by rifle bullets are discussed in the light of the most recent experience of the Japanese, Russian and German surgeons in the Manchurian War. Matas' article extends to 350 pages, and contains a large amount of new material not to be found in any other text-book. No surgeon can afford to neglect this very important work.

The remaining chapters in the volume are distributed as follows:- 